\title{
11th International Conference on Geometry and Applications
}

\author{
Varna (Bulgaria), September 1-6, 2013
}

The conference took place in the International Home of Scientists "F. JoliotCurie", St. Constantine and Helena, Bulgaria. The Scientific Committee which led the conference consisted of W. Benz, R. Fritsch, H. Havlicek, S. Ivanov, H. Karzel, A. Kreuzer, H.-J. Kroll, Ch. Lozanov, M. Marchi, D. Mekerov, U. Simon, G. Stanilov (Chairman), N. Stephanidis, and H. Wefelscheid. Laudations in honour of Hans Havlicek, Udo Simon, and Dirk Wefelscheid were given by Rolf Riesinger, Heinrich Wefelscheid, and Helmut Karzel, respectively. A talk in memoriam Werner Heise (1944-2013) was given by Ivan Landjev. The following are abstracts of the scientific talks presented at the conference.

Danish Ali (GC University, Lahore, Pakistan)

Johann Davidov (Bulgarian Academy of Sciences, Bulgaria)

Oleg Mushkarov (Bulgarian Academy of Sciences, Bulgaria)

\section{Holomorphic Curvatures of Twistor Spaces}

We study the twistor spaces of oriented Riemannian four-manifolds as a source of almost Hermitian six-manifolds of constant or strictly positive holomorphic, Hermitian and orthogonal bisectional curvatures. In particular, we obtain explicit formulas for these curvatures in the case when the base manifold is Einstein and self-dual and observe that the "squashed" metric on $\mathbb{C P}^{3}$ is a non-Kähler Hermitian-Einstein metric of positive holomorphic bisectional curvature. This shows that a recent result of Kalafat and Koca in dimension four cannot be extended to higher dimensions. We prove that the Hermitian bisectional curvature of a non-Kähler Hermitian manifold is never a non-zero constant which gives a partial negative answer to a question of Balas and Gauduchon. Finally, motivated by an integrability result of Vezzoni for almost Kähler manifolds, we study the problem when the holomorphic and the Hermitian bisectional curvatures of an almost Hermitian manifold coincide. 
We extend the result of Vezzoni to a more general class of almost Hermitian manifolds and describe the twistor spaces having this curvature property.

Seher Aslanci (Ordu University, Turkey)

Rabia Cakan (Ataturk University, Turkey)

\section{On a Cotangent Bundle with Deformed Riemannian Extension}

Let $\left(M_{n}, g\right)$ be a Riemannian manifold with metric $g$ and ${ }^{C} T\left(M_{n}\right)$ be its cotangent bundle with local coordinates $\left(\pi^{-1}(U), x^{i}, x^{\bar{i}}=p_{i}\right), i=1, \ldots, n$; $\bar{i}=n+i=n+1, \ldots, 2 n ; U \subset M_{n}$. A new (pseudo) Riemannian metric $\nabla_{g} \in \mathfrak{T}_{2}^{0}\left({ }^{C} T\left(M_{n}\right)\right)$ on ${ }^{C} T\left(M_{n}\right)$ is defined by the equation

$$
\nabla_{g}\left({ }^{C} X,{ }^{C} Y\right)=-\gamma\left(\nabla_{X} Y+\nabla_{Y} X\right)
$$

for any $X, Y \in \mathfrak{T}_{0}^{1}\left(M_{n}\right)$, where $\gamma\left(\nabla_{X} Y+\nabla_{Y} X\right)$ is a function in $\pi^{-1}(U) \subset$ ${ }^{C} T\left(M_{n}\right)$ with local expression $\gamma\left(\nabla_{X} Y+\nabla_{Y} X\right)=p_{h}\left(X^{i} \nabla_{i} Y^{h}+Y^{i} \nabla_{i} X^{h}\right)$. We call $\nabla_{g}$ the Riemannian extension of the Levi-Civita connection $\nabla_{g}$ to ${ }^{C} T\left(M_{n}\right)$. The main purpose of this paper is to study deformed Riemannian extensions $\nabla_{g}+{ }^{V} g$ in the cotangent bundle. The curvature properties of metric connections for deformed Riemannian extensions are also investigated.

The second author was supported by The Scientific and Technological Research Council of Turkey (TBAG-1001, Project No. 112T111).

\section{Dieter Betten (University of Kiel, Germany) Rolf Riesinger (Vienna, Austria)}

\section{Automorphic Collineations of Regular Parallelisms of PG(3, $\mathbb{R})$}

A parallelism of a projective 3 -space $\Pi$ is a family $\mathbf{P}$ of spreads such that each line of $\Pi$ is contained in exactly one spread of $\mathbf{P}$. A parallelism of the real projective 3 -space $\mathrm{PG}(3, \mathbb{R})$ is called topological, if the operation of drawing a line parallel to a given line through a given point is continuous. Let $\lambda$ be Klein's correspondence and $\pi_{5}$ the polarity associated with the Klein quadric $H_{5}$. If $\mathcal{C}$ is a regular spread, then $\lambda(\mathcal{C})$ is an elliptic subquadric of $H_{5}$ which is contained in the 3 -space $\operatorname{span} \lambda(\mathcal{C})$ and the line $\pi_{5}(\operatorname{span} \lambda(\mathcal{C}))$ is a 0 -secant of $H_{5}$. Let $\mathbf{C}$ be the set of all regular spreads of $\mathrm{PG}(3, \mathbb{R})$ and let $\mathbf{Z}$ be the set of all lines of $\operatorname{PG}(5, \mathbb{R})$ which are 0 -secants of $H_{5}$. Then $\gamma: \mathbf{C} \rightarrow \mathbf{Z} ; \mathcal{C} \mapsto \pi_{5}(\operatorname{span} \lambda(\mathcal{C}))=: \gamma(\mathcal{C})$ is a bijective map. The $\gamma$-image of the Clifford parallelism is a plane of lines. To each collineation or duality $\tau$ of the extended projective group $\operatorname{PGL}_{e}(4, \mathbb{R})$ there belongs by virtue of Klein's correspondence $\lambda$ of line geometry an induced collineation $\tau_{\lambda}$ of the group $A$ ut $H_{5}$ of all collineations of $\operatorname{PGL}(6, \mathbb{R})$ leaving the Klein quadric $H_{5}$ invariant. The mapping $\tau \mapsto \tau_{\lambda}$ is a homeomorphism from $\mathrm{PGL}_{e}(4, \mathbb{R})$ onto 
Aut $H_{5}$. A parallelism of $\mathrm{PG}(3, \mathbb{R})$ all whose members are regular spreads is called regular. For a regular parallelism $\mathbf{T}$ of $\mathrm{PG}(3, \mathbb{R})$ we put

$$
\gamma(\mathbf{T}):=\{\gamma(\mathcal{C}) \mid \mathcal{C} \in \mathbf{T}\} \quad \text { and } \operatorname{dim}(\operatorname{span} \gamma(\mathbf{T}))=: d_{\mathbf{T}}
$$

We call $d_{\mathbf{T}}$ the dimension of $\mathbf{T}$. Clifford and 2-dimensional parallelisms coincide.

Let $\mathbf{P}$ be a topological parallelism $\mathrm{PG}(3, \mathbb{R})$ and denote by $\Gamma$ the group of all automorphic collineations of $\mathbf{P}$. The homogeneity of a topological parallelism $\mathbf{P}$ is measured by the (topological) dimension of the connected component, say $\Delta_{\mathbf{P}}$, of the identity in $\Gamma$. If $\operatorname{dim} \Delta_{\mathbf{P}}=g$, then we say that $\mathbf{P}$ is of group dimension $g$.

1. There exists no parallelism $\mathbf{P}$ with $\operatorname{dim} \Delta_{\mathbf{P}} \geq 7$.

2. If $\operatorname{dim} \Delta_{\mathbf{P}}=6$, then $\mathbf{P}$ is a Clifford parallelism.

3. There exists no parallelism $\mathbf{P}$ with $\operatorname{dim} \Delta_{\mathbf{P}}=5$.

Under the latent line set of a regular parallelism $\mathbf{T}$ of $\mathrm{PG}(3, \mathbb{R})$ we understand

$$
\lambda^{-1}\left(\operatorname{span} \gamma(\mathbf{T}) \cap H_{5}\right)=: \Lambda_{\mathbf{T}} \subseteq \mathcal{L}_{3} .
$$

The latent line set $\Lambda_{\mathbf{T}}$ of a regular 3-dimensional parallelism $\mathbf{T}$ is a regular spread which is no member of $\mathbf{T}$.

If the regular parallelism $\mathbf{Z}$ of $\operatorname{PG}(3, \mathbb{R})$ is constructed via the hyperflock determining lineset $\mathcal{Z}$, then the group $A u t_{e} \mathbf{Z}$ of all automorphic collineations and dualities of $\mathbf{Z}$ is isomorphic to the group Aut $H_{5} \cap$ Aut $\mathcal{Z}$.

\section{References}

[1] Betten, D., Riesinger, R.: Hyperflock determining line sets and totally regular parallelisms of $\mathrm{PG}(3, \mathbb{R})$. Mh. Math. 161, 43-58 (2010).

[2] Betten, D., Riesinger, R.: Collineation groups of topological parallelisms. Adv. Geom. (to appear).

Iva Dokuzova (University of Plovdiv, Bulgaria)

Dimitar Razpopov (Agricultural University of Plovdiv, Bulgaria)

Georgi Dzhelepov (Plovdiv, Bulgaria)

\section{Three-Dimensional Riemannian Manifolds with Circulant Structures}

The main purpose of the present paper is to continue the considerations in [1] and to get more general results. We consider a 3-dimensional Riemannian manifold $M$ with a metric $g$ and affinor structure $q$. We suppose there exists a local coordinate system, where $g$ and $q$ have circulant coordinates:

$$
\left(g_{i j}\right)=\left(\begin{array}{lll}
A & B & B \\
B & A & B \\
B & B & A
\end{array}\right), \quad\left(q_{i}^{s}\right)=\left(\begin{array}{lll}
0 & 1 & 0 \\
0 & 0 & 1 \\
1 & 0 & 0
\end{array}\right) .
$$


The functions $A=A(p), B=B(p) \in F M, p \in M$ satisfy $A>B>0$. We have $q^{3}=E, g(q x, q y)=g(x, y), x, y \in \chi M$, where $E$ is the unit matrix [1]. If $M$ satisfies the above conditions, we write that $M$ is in a class $V$.

If $M \in V$ satisfies $R(q x, q y, q z, q u)=R(x, y, z, u), x, y, z, u \in \chi M$, then $M$ is in a class $V_{2}$. We prove the next assertions:

1. The class $V_{2}$ is not empty.

2. If $M$ is in $V_{2}$ and $x$ is an arbitrary vector in $T_{p} M$, then the sectional curvatures of 2 -sections $\{x, q x\},\left\{q x, q^{2} x\right\},\left\{x, q^{2} x\right\}$ are equal.

3. Let $M$ be in $V_{2},\left\{x, q x, q^{2} x\right\}$ be an orthonormal $q$-base in $T_{p} M$ and $\angle(y, q y)=\frac{\pi}{3}, y \in T_{p} M$. If $u$ is an arbitrary vector in $T_{p} M$, then the sectional curvature $\mu(u, q u)$ of 2 -section $\{u, q u\}$ is a monotone function of the angle $\varphi=\angle(u, q u)$ and

$$
\mu(u, q u)=\frac{1-2 \cos \varphi}{1+2 \cos \varphi} \mu(x, q x)+\frac{3 \cos \varphi}{1+2 \cos \varphi} \mu(y, q y), \varphi \in\left(0, \frac{2 \pi}{3}\right) .
$$

The author is supported by project NI13-FMI-002 of the Scientific Research Fund, Paisii Hilendarski University of Plovdiv, Bulgaria.

\section{References}

[1] Dzhelepov, G., Dokuzova, I., Razpopov, D.: On a three-dimensional Riemannian manifold with an additional structure. PU Sci. Works Math.38(3), 17-27 (2011). arXiv:math.DG/ 0905.0801 .

[2] Borisov, A., Ganchev, G.: Curvature properties of Kaehlerian manifolds with B-metric. Math. Educ. Math., Proceedings of the 14th Spring Conference UBM, Sunny Beach, pp. 220-226 (1985).

[3] Grey, A.: Curvature identities for Hermitian and Almost Hermitian Manifolds. Tohoku Math. J. 28(4), 601-612 (1976).

[4] Yano, K.: Differential geometry on complex and almost complex spaces. Pure Appl. Math., vol. 49. Pergamon press, New York (1965).

\section{Radostina Encheva (Konstantin Preslavsky University of Shumen, Bulgaria)}

\section{Möbius Invariants of Space Curves}

We consider the unit hypersphere $S^{3}$, centered at the origin, embedded in four-dimensional Euclidean space $\mathbb{E}^{4}$. The stereographic projection $\pi$ from the north pole $P \in S^{3}$ onto the equatorial hyperplane of $S^{3}$, provided with a point at infinity $\infty$, is a bijective conformal map that maps the punctured sphere $S^{3} \backslash P$ in a three-dimensional space $\mathbb{R}^{3}$. If $S O(4)$ is the group of rotations in $\mathbb{E}^{4}$, preserving the sphere $S^{3}$, then $S O(4)$ coincides with the group of rigid motions on the sphere $S^{3}$. This group induces in $\mathbb{R}^{3}$ via the stereographic projection $\pi$ a subgroup $F_{0}$ of the Möbius group Möb(3). The group of rotations $S O(4)$ and the 3 -sphere $S^{3}$ have a natural representation by the algebra $\mathbb{H}$ of all 
quaternions. We can identify the points of the sphere $S^{3}$ with the set of all unit quaternions. In [2], the Cayley's theorem describes the group $S O(4)$ by pairs of unit quaternions. For every pair $(a, b) \in S^{3} \times S^{3}$ there exists an orthogonal transformation $\varphi(a, b): \mathbb{H} \rightarrow \mathbb{H}, x \mapsto a \cdot x \cdot b$ and $\varphi\left(S^{3} \times S^{3}\right)=S O(\mathbb{H})$. Since $\mathbb{H}$ and $\mathbb{E}^{4}$ are isomorphic Euclidean spaces, their groups of rotations $S O(\mathbb{H})$ and $S O(4)$ are isomorphic and we will identify them. The points of the equatorial hyperplane of $S^{3}$ can be represented by the set $\mathbb{H}_{0}$ of pure quaternions. A pure quaternion is one of zero real part. Using the very simple presentation of the stereographic projection $\pi$ by quaternions in [1], the transformation of the group $F_{0} \subset \operatorname{Möb}(3)$ can be expressed by the quaternionic formalism

$$
f(q)=\frac{q+1+a(q-1) b}{q+1-a(q-1) b}, \quad q \in \mathbb{H}_{0}, a, b \in S^{3} .
$$

In [3], Tazawa interprets the Euclidean theory of the curves and the surfaces in $\mathbb{R}^{3}$ on the 3 -sphere $S^{3}$ in $\mathbb{R}^{4}$. Any spherical curve $\gamma$ on the unit sphere $S^{3}$, parameterized by an arc length parameter $\sigma$, is defined up to a rigid motion on the sphere by the functions $\tilde{\varkappa}$ and $\tilde{\tau}$, called "spherical curvature" and "spherical torsion" respectively. If $\varkappa$ and $\tau$ are the Euclidean curvature and the Euclidean torsion of the stereographic image $c=\pi(\gamma)$ of $\gamma$, with arc length function $s=s(\sigma)$, then we obtain that

$$
\tilde{\varkappa}^{2}(s)=\frac{1}{\mu} \varkappa^{2}(s)-1-\frac{2}{\mu} S_{\sigma}(s), \quad \tilde{\tau}(s)=\frac{\sin \theta}{\mu \tilde{\varkappa}} \varkappa^{\prime}-\frac{\cos \theta}{\mu \tilde{\varkappa}} \varkappa \tau,
$$

where $S_{\sigma}(s)$ is the Schwarzian derivative of the arc length function $\sigma=\sigma(s)$, $\mu$ is the non-zero component of the metric tensor $g$ of $S^{3}$ in a parametrization given by the stereographic projection $\pi$. These functions are invariants under the group $F_{0} \mathrm{Möb}(3)$. We prove theorem that determines any space curve up to transformation of the group $F_{0}$. Applying the computer system Mathematica, we give an algorithm of recovering a space curve from its invariants under $F_{0}$.

\section{References}

[1] Georgiev, G.H.: Geometric Transformations for Modeling of Curves and Surfaces. Shaker Verlag, Aachen (2012).

[2] Koecher, M., Remmert, R.: Hamilton's Quaternions. In: Ewing, J.H. (ed.) Numbers Graduate Text in Mathematics, vol. 123, Springer, New York, pp. 189-220 (1991).

[3] Tazawa, Y.: Curves and surfaces in the three dimensional sphere placed in the space of quaternions, Innovation in mathematics. Trans. Eng. Sci. 15, 459-466 (1997).

\section{Georgi H. Georgiev (Konstantin Preslavsky University, Bulgaria)}

\section{Plane and Space Spirals}

The similarity transformations preserve the sets of spirals in the Euclidean plane and the Euclidean 3-space. Differential geometric invariants of curves with respect to the similarity transformations are known as shape curvatures. 
In this talk, we present some relationships between spirals and shape curvatures In particular, Kurnosenko's classification of plane spirals and applications of short spirals in geometric modeling are discussed. Main classes of space spirals are described in terms of shape curvatures.

\section{References}

[1] Encheva, R., Georgiev, G.: Shapes of space curves. J. Geom. Graph. 7, 145-155 (2003).

[2] Encheva, R., Georgiev, G.: Similar Frenet curves, result. Math. 55(3-4), 359-372 (2009).

[3] Georgiev, G.H.: Shape Curvatures of planar rational spirals. Lect. Notes Comput Sci. 6920, 270-279 (2012).

[4] Goodman, T.N.T., Meek, D.S., Walton, D.J.: An involute spiral that matches G2 Hermite data in the plane. Comp. Aided Geom. Design 26,733-756 (2009).

[5] Kurnosenko, A.I.: Short spirals. J. Math. Sci. (NY) 175(5), 517-522 (2011)

\section{Sava Grozdev (Bulgarian Academy of Sciences, Bulgaria) Veselin Nenkov (Technical College Lovech, Bulgaria)}

\section{A property of Simson Lines in a Quadrilateral}

The well-known Simson line in the geometry of the triangle could be generalized in the following way. For an arbitrary $\triangle A B C$ let $M_{a}, M_{b}$ and $M_{c}$ be the mid-points of the segments $B C, C A$ and $A B$, respectively, while $k(O)$ be an ellipse or a hyperbola with center $O$, which is circumscribed round $\triangle A B C$. If $P$ is an arbitrary point in the plane of $\triangle A B C$, construct the lines $p_{a}, p_{b}$ and $p_{c}$ through $P$, which are parallel to $O M_{a}, O M_{b}$ and $O M_{c}$, respectively. By the lines $p_{a}, p_{b}$ and $p_{c}$ define the points $P_{a}=p_{a} \cap B C, P_{b}=p_{b} \cap C A$ and $P_{c}=p_{c} \cap A B$. The points $P_{a}, P_{b}$ and $P_{c}$ are collinear iff $P$ is on $k(O)$. The line $s_{P}$ through $P_{a}, P_{b}$ and $P_{c}$ is called Simson line of the point $P$ with respect to $\triangle A B C$ and the conic $k(O)$.

An interesting property of Simson lines is included in the following

Theorem. If $A B C D$ is a quadrilateral, which is inscribed in an ellipse or hyperbola $k(O)$, then the Simson lines of the points $A, B, C$ and $D$ with respect to the triangles $B C D, C D A, D A B$ and $A B C$ have a common point $H$.

When $k(O)$ is a circle, then the point $H$ is called orthocenter of the quadrilateral $A B C D$. Thus, from the theorem we get the following

Corollary. If the quadrilateral $A B C D$ is cyclic, then the Simson lines of $A, B$, $C$ and $D$ with respect to the triangles $B C D, C D A, D A B$ and $A B C$ respectively intersect in the orthocenter of $A B C D$.

It turns out that some remarkable conics in connection with the quadrilateral $A B C D$ pass through the point $H$. 


\section{Hans Havlicek (Vienna University of Technology, Austria)}

\section{A Note on Segre Varieties in Characteristic Two}

Let $\boldsymbol{V}_{1}, \boldsymbol{V}_{2}, \ldots, \boldsymbol{V}_{m}$ be $m \geq 1$ two-dimensional vector spaces over the same commutative field $F$. The non-zero decomposable tensors of $\bigotimes_{k=1}^{m} \boldsymbol{V}_{k}$ determine the Segre variety $\mathcal{S}_{(m)}(F)$ in the projective space $\mathbb{P}\left(\bigotimes_{k=1}^{m} \boldsymbol{V}_{k}\right)$. Each $\boldsymbol{V}_{k}$ admits a symplectic bilinear form $[\cdot, \cdot]$, so that $\bigotimes_{k=1}^{m} \boldsymbol{V}_{k}$ is equipped with a bilinear form which is given by

$$
\left[\boldsymbol{a}_{1} \otimes \boldsymbol{a}_{2} \otimes \cdots \otimes \boldsymbol{a}_{m}, \boldsymbol{b}_{1} \otimes \boldsymbol{b}_{2} \otimes \cdots \otimes \boldsymbol{b}_{m}\right]:=\prod_{k=1}^{m}\left[\boldsymbol{a}_{k}, \boldsymbol{b}_{k}\right] \quad \text { for } \boldsymbol{a}_{k}, \boldsymbol{b}_{k} \in \boldsymbol{V}_{k} .
$$

In projective terms this form yields the well known fundamental polarity of $\mathcal{S}_{(m)}(F)$. It is associated to a regular quadric when $m$ is even and Char $F \neq 2$. Otherwise it is a null polarity.

In our talk we focus on the case when $F$ has characteristic two. Here the fundamental polarity of $\mathcal{S}_{(m)}(F)$ is always null, and there exists an invariant quadric:

Theorem. Let $m \geq 2$ and Char $F=2$. There exists in the ambient space of the Segre $\mathcal{S}_{(m)}(F)$ a regular quadric $\mathcal{Q}(F)$ with the following properties:

1. The projective index of $\mathcal{Q}(F)$ is $2^{m-1}-1$.

2. $\mathcal{Q}(F)$ is invariant under the group of projective collineations stabilising the Segre $\mathcal{S}_{(m)}(F)$.

This is joint work with Boris Odehnal (Vienna, Austria) and Metod Saniga (Tatranská Lomnica, Slovakia).

\section{Reference}

[1] Havlicek, H., Odehnal, B., Saniga, M.: On invariant notions of Segre varieties in binary projective spaces. Des. Codes Cryptogr. 62, 343-356 (2012)

Stefan Ivanov (University of Sofia, Bulgaria)

Alexander Petkov (University of Sofia, Bulgaria)

Dimiter Vassilev (University of New Mexico, New Mexico, USA)

\section{The Obata Sphere Theorems on a Quaternionic Contact Manifold of Dimension Bigger than Seven}

Using the classical Bochner-Weitzenböck formula Lichnerowicz [5] showed that on a compact Riemannian manifold $(M, h)$ of dimension $n$ for which the Ricci curvature is greater than or equal to that of the round unit $n$-dimensional sphere $S^{n}(1)$, the first positive eigenvalue $\lambda_{1}$ of the Laplace operator is greater than or equal to the first eigenvalue of the sphere, $\lambda_{1} \geq n$. Subsequently Obata [6] proved that equality is achieved if and only if the Riemannian manifold 
is isometric to $S^{n}(1)$ by noting that the trace-free part of the Riemannian Hessian of an eigenfunction $f$ with eigenvalue $\lambda=n$ vanishes, i.e., it satisfies the system

$$
\left(\nabla^{h}\right)^{2} f=-f h .
$$

In fact, Obata showed that on a complete Riemannian manifold $(M, h)$ equation (1) allows a non-constant solution if and only if the manifold is isometric to the unit sphere. In this case, the eigenfunctions corresponding to the first eigenvalue are the solutions of (1).

We prove quaternionic contact versions of two of Obata's sphere theorems.

Theorem 1. ([3]) Let $(M, \eta, g, \mathbb{Q})$ be a compact quaternionic contact $(q c)$ manifold of dimension bigger than seven whose Ricci tensor satisfies the Lichnerowicz type lower bound estimate ([2]). Then the first positive eigenvalue of the sub-Laplacian takes the smallest possible value [2] if and only if the qc manifold is qc equivalent to the standard 3-Sasakian sphere.

Theorem 1 follows from the following

Theorem 2. ([3]) Let $(M, \eta, g, \mathbb{Q})$ be a quaternionic contact manifold of dimension $4 n+3>7$ which is complete with respect to the associated Riemannian metric $h=g+\left(\eta_{1}\right)^{2}+\left(\eta_{2}\right)^{2}+\left(\eta_{3}\right)^{2}$.

Suppose there exists a non-constant smooth function $f$ whose horizontal Hessian satisfies

$$
\nabla d f(X, Y)=-f g(X, Y)-\sum_{s=1}^{3} d f\left(\xi_{s}\right) \omega_{s}(X, Y),
$$

where $\nabla$ is the Biquard connection.

Then the qc manifold $(M, \eta, g, \mathbb{Q})$ is qc homothetic to the unit $(4 n+3)$ dimensional 3-Sasakian sphere.

The work relies on considerations in [1]. We achieve the proof of Theorem 2 by showing first that $M$ is isometric to the unit sphere $S^{4 n+3}$ and then that $M$ is qc-equivalent to the standard 3-Sasakian structure on $S^{4 n+3}$. To this effect we show that the torsion of the Biquard connection vanishes and in this case the Riemannian Hessian satisfies (1) after which we invoke the classical Obata theorem showing that $M$ is isometric to the unit sphere. In order to prove the qc-equivalence part we show that the qc-conformal curvature vanishes, which gives the local qc conformal equivalence with the 3-Sasakian sphere due to [4, Theorem 1.3], and then prove a Liouville-type result, which implies the existence of a global qc-conformal map between $M$ and the 3-Sasakian sphere.

\section{References}

[1] Ivanov, S., Minchev, I., Vassilev, D.: Quaternionic contact Einstein structures and the quaternionic contact Yamabe problem. Memoirs Am. Math. Soc. (to appear).

[2] Ivanov, S., Petkov, A., Vassilev, D.: The sharp lower bound of the first eigenvalue of the sub-Laplacian on a quaternionic contact manifold. J. Geom. Anal. doi:10.1007/ s12220-012-9354-9. arXiv:1112.0779. 
[3] Ivanov, S., Petkov, A., Vassilev, D.: The Obata sphere theorems on a quaternionic contact manifold of dimension bigger than seven. arXiv:1303.0409 (submitted).

[4] Lichnerowicz, A.: Géométrie des groupes de transformations. Travaux et Recherches Mathematiques, vol. III. Dunod, Paris (1958).

[5] Obata, M.: Certain conditions for a Riemannian manifold to be isometric with a sphere. J. Math. Soc. Jpn 14 (3), 333-340 (1962).

\section{Alexander B. Kharazishvili (Technical University, Tbilisi, Georgia) \\ On Three-Colorings of the Euclidean Plane and Associated Triangles of a Prescribed Type}

A three-coloring of the Euclidean plane $R^{2}$ is defined as any surjection $\mathbf{g}$ of $R^{2}$ onto the three-element set $\{1,2,3\}$.

A triangle $[x, y, z]$ in $R^{2}$, whose vertices are $x, y, z$, is said to be associated with a three-coloring $\mathbf{g}$ (in short, $\mathbf{g}$-associated) if $\mathbf{g}(\{x, y, z\})=\{1,2,3\}$.

The question naturally arises: how many triangles of a prescribed type (e.g., acute, obtuse, right, isosceles) can be associated with $\mathbf{g}$ ?

Theorem 1. For any three-coloring $\mathbf{g}$ of $R^{2}$, there exist:

(a) continuum many g-associated triangles, all of which are acute-angled;

(b) continuum many g-associated triangles, all of which are obtuse-angled;

(c) continuum many g-associated triangles, all of which are right-angled;

(d) continuum many g-associated triangles, all of which are isosceles.

On the other hand, we have the following statement.

Theorem 2. For any given triangle $[x, y, z]$ in the plane $R^{2}$, there exists a threecovering $\mathbf{g}$ of $R^{2}$ such that no triangle in $R^{2}$ similar to $[x, y, z]$ is $\mathbf{g}$-associated.

Theorem 2 can be generalized to the case of a multi-dimensional Euclidean space, while Theorem 1 does no admit such a generalization.

Aleks Kirtadze (Georgian Technical University, Tbilisi, Georgia) Tamar Kasrashvili (Georgian Technical University, Tbilisi, Georgia)

\section{Elementary Volume from the Measure-Theoretical View-Point}

The concept of volume for sufficiently simple geometric figures is one of the most important in geometry of Euclidean space. A discussion of this concept occupies a substantial place in standard university lecture courses of geometry (see, e.g., [1]). This deep notion is closely connected with several interesting 
and important geometric topics, such as equi-decomposability theory (including well-known paradoxes), dissections of figures into finitely many ones of a prescribed type, the behavior of the volume function under Minkowski's sum of point sets, etc. One of the principal problems which arises here is to extend the function of elementary volume to a volume defined on a maximally large class of figures. This problem is successfully solved within framework of modern theory of invariant measures and its solution heavily depends on purely algebraic properties of a basic group of transformations of the Euclidean space. The present report is devoted to some aspects highlighting tight connections between elementary theory of volume with general methods of the theory of invariant measures.

\section{Reference}

[1] Hadwiger, H.: Vorlesungen über Inhalt, Oberfläche und Isoperimetrie. Springer-Verlag, Berlin (1957).

Ivan Landjev (New Bulgarian University, Sofia, Bulgaria)

\section{Sperner's Theorem for Modules Over Finite Chain Rings}

Let $R$ be a finite chain ring with $|R|=q^{m}, R / \operatorname{rad} R \cong F_{q}$. Every submodule $M$ of ${ }_{R} R^{n}$ is isomorphic to a direct sum of cyclic modules:

$$
M \cong R / N^{\lambda_{1}} \oplus R / N^{\lambda_{2}} \oplus \cdots \oplus R / N^{\lambda_{n}},
$$

where $\lambda_{1} \geq \lambda_{2} \geq \ldots \geq \lambda_{n} \geq 0$ are integers and $N=\operatorname{rad} R$. The $n$-tuple $\left(\lambda_{1}, \lambda_{2}, \ldots, \lambda_{n}\right)$ is called the shape of $M$. We consider the partially ordered set $\mathcal{P}$ of all submodules contained in a module of shape $\lambda=\left(\lambda_{1}, \ldots, \lambda_{n}\right)$. We prove an analogue of Sperner's theorem saying that the size of a maximal antichain in $\mathcal{P}$ is equal to

$$
\sum_{\mu \prec \lambda}\left[\begin{array}{l}
\lambda \\
\mu
\end{array}\right]_{q^{m}}
$$

where $\left[\begin{array}{l}\lambda \\ \mu\end{array}\right]_{q^{m}}$ denotes the number of all modules of shape $\mu$ contained in a module of shape $\lambda$ and the sum is over all partitions $\mu=\left(\mu_{1}, \ldots, \mu_{n}\right) \prec \lambda$ with $\sum_{i} \mu_{i}=\left\lfloor\left(\sum_{i} \lambda_{i}\right) / 2\right\rfloor$.

Hristo Manev (Plovdiv Medical University, Bulgaria)

\section{The Components of the Structure Tensor of Almost Contact Manifolds with B-Metric}

The present work deals with differential geometry of almost contact manifolds with B-metric. These manifolds are the odd-dimensional extension of almost 
complex manifolds with Norden metric and an almost neutral-signature analogue of almost contact manifolds with compatible metric. The differential geometry of the considered manifolds are developed but some important aspects are still obscure.

The almost contact endomorphism $\varphi$ is an almost complex structure on the contact distribution $\operatorname{ker}(\eta)$ and $\varphi$ acts as an anti-isometry with respect to the restriction of the B-metric $g$ on $\operatorname{ker}(\eta)$. In distinction from the Hermitian case, the basic B-metric generates an associated B-metric (not a Kähler form).

Our main goal is to classify the almost contact manifolds with B-metric of dimension three - the smallest dimension of the manifold under consideration.

We consider the structure tensor $F$ generated by the covariant derivative of the almost contact endomorphism with respect to the Levi-Civita connection of the basic B-metric. There is known a classification regarding $F$ made by $\mathrm{G}$. Ganchev, V. Mihova and K. Gribachev in 1993. Following this classification, we determine the components of $F$ in each of the eleven basic classes for the odd dimension in general.

The case of the smallest dimension 3 is object of special interest in our consideration. We determine which of the eleven basic classes of the almost contact manifolds with B-metric are empty in the case of dimension 3.

We adduce appropriate examples of the considered manifolds of dimension 3.

This work is partially supported by project NI13-FMI-002 of the Scientific Research Fund, Plovdiv University, Bulgaria.

\section{Mancho Manev (Paisii Hilendarski University of Plovdiv, Bulgaria)}

\section{Hypercomplex Structures with Hermitian-Norden Metrics on Four-Dimensional Lie Algebras}

The present work is inspired by the work of Barberis ([1]) where invariant hypercomplex structures $H$ on 4-dimensional real Lie groups are classified. In that case the corresponding metric is positive definite and Hermitian with respect to the triplet of complex structures of $H$. Our main goal is to classify 4-dimensional real Lie algebras which admit hypercomplex structures with Hermitian and Norden metrics.

We equip a hypercomplex structure $H$ with a metric structure, generated by a pseudo-Riemannian metric $g$ of neutral signature. In our case the one (resp., the other two) of the almost complex structures of $H$ acts as an isometry (resp., act as anti-isometries) with respect to $g$ in each tangent fibre.

Thus, there exist three $(0,2)$-tensors associated by $H$ except the metric $g-\mathrm{a}$ Kähler form and two metrics of the same type. 
The metric $g$ is Hermitian with respect to the one almost complex structure of $H$ and $g$ is a Norden (i.e. an anti-Hermitian) metric regarding the other two almost complex structures of $H$. For this reason we call the derived almost hypercomplex structure an almost hypercomplex structure with Hermitian-Norden-metrics.

The corresponding five types of invariant hypercomplex structures with hyperHermitian metric, studied by M.L. Barberis, are constructed here. The different cases regarding the signature of the basic pseudo-Riemannian metric are considered.

This work is partially supported by project NI13-FMI-002 of the Scientific Research Fund, Plovdiv University, Bulgaria and the German Academic Exchange Service $(D A A D)$

\section{Reference}

[1] Barberis, M.L.: Hypercomplex structures on four-dimensional Lie groups. Proc. Am. Math. Soc. 125(4), 1043-1054 (1997)

\section{Mancho Manev (Paisii Hilendarski University of Plovdiv, Bulgaria)}

\section{Miroslava Ivanova (Trakia University, Stara Zagora, Bulgaria)}

\section{Almost Contact B-Metric Manifolds with Curvature Tensors of Kähler Type}

In this work we prove that the any $\varphi$-Kähler-type tensor (i.e. a tensor satisfying the properties of the curvature tensor of the Levi-Civita connection in the special class of the parallel structures on the manifold) on 5-dimensional almost contact B-metric manifold $(M, \varphi, \xi, \eta, g)$ is a linear combination of two known tensors of the same type, generated by the metric tensors of $(M, \varphi, \xi, \eta, g)$ as the coefficients are the sectional curvature of the totally real 2-planes $\xi$ orthogonal with respect to this tensor. We find the expression of the associated 1-forms by the scalar curvatures of the $\varphi$-Kähler-type tensor for the $\varphi$-canonical connection on the manifolds from the main classes with closed 1-forms. Some of obtained results we illustrated by a known example of an almost contact B-metric manifold given in [1].

This work is partially supported by project NI11-FMI-004 of the Scientific Research Fund, Paisii Hilendarski University of Plovdiv, Bulgaria.

\section{Reference}

[1] Ganchev, G., Mihova, V., Gribachev, K.: Almost contact manifolds with B-metric. Math. Balkanica (N.S.), 7(3-4), 261-276 (1993). 
Galya Nakova (University of Veliko Tarnovo St. Cyril and St. Methodius, Bulgaria)

\section{On Some Classes of Lightlike Submanifolds and Hypersurfaces of Almost Complex Manifolds with Norden Metric}

The general theory of lightlike submanifolds has been developed by K. Duggal and A. Bejancu. Many new types of lightlike submanifolds of indefinite Kaehler manifolds, indefinite Sasakian and indefinite quaternion Kaehler manifolds are introduced by K. Duggal and B. Sahin. I study lightlike submanifolds of almost complex manifolds with Norden metric (or $B$-metric) which have not been considered yet.

Let $M$ be a real $m$-dimensional submanifold of an almost complex manifold with Norden metric $(\bar{M}, \bar{J}, \bar{g}, \overline{\widetilde{g}})$. The geometry of $M$ depends on the behaviour of the tangent bundle of $M$ with respect to the action of the almost complex structure $\bar{J}$ and the induced metric on $M$. Due to the fact that there exist two Norden metrics $\bar{g}$ and $\overline{\widetilde{g}}$ of $\bar{M}$ we can consider two induced metrics $g$ and $\widetilde{g}$ on $M$ by $\bar{g}$ and $\overline{\widetilde{g}}$, respectively. For a submanifold $M$ of $\bar{M}$ three cases with respect to the induced metrics $g$ and $g$ on $M$ are possible: $M$ is nondegenerate with respect to both $g$ and $\widetilde{g} ; M$ is degenerate with respect to both $g$ and $\widetilde{g}$; $\mathrm{M}$ is non-degenerate with respect to $g$ (resp. $\widetilde{g}$ ) and degenerate with respect to $\widetilde{g}$ (resp. $g$ ). I consider submanifolds of the third type and prove that the submanifold $(M, g)$ of a $2 n$-dimensional almost complex manifold with Norden metric $(\bar{M}, \bar{J}, \bar{g}, \overline{\widetilde{g}})$ is a CR-submanifold with an $r$-dimensional totally real distribution $D^{\perp}$ if and only if $(M, \widetilde{g})$ is an $r$-lightlike Radical transversal lightlike submanifold. Relations between the induced geometric objects of some of these submanifolds are obtained. I introduce radical transversal lightlike hypersurfaces of almost complex manifolds with Norden metric and give some geometrical characterizations of them. Examples of the considered lightlike submanifolds and hypersurfaces are constructed.

\section{Alexander Petkov (University of Sofia, Bulgaria) \\ Stefan Ivanov (University of Sofia, Bulgaria) \\ Dimiter Vassilev (University of New Mexico, New Mexico, USA) \\ The Sharp Lower Bound of the First Eigenvalue of the Sub-Laplacian on a Quaternionic Contact Manifold}

In this talk we give analogues of the classical theorems of Lichnerowicz and Obata in the case of quaternionic contact manifolds. The Lichnerowicz-type theorem [1] says, that under some condition imposed on the Ricci tensor and the torsion tensor of the Biquard connection of a compact quaternionic contact manifold, it is obtained a sharp lower bound of the first eigenvalue of the sub-Laplacian. The estimate is sharp in the sense, that the equality in the 
corresponding inequality is obtained on the 3-Sasakian sphere. To obtain the estimate on the seven dimensional quaternionic contact manifolds, we need by an extra condition, concerning the non-negativity of the $P$-function of any eigenfunction [2].

The Obata-type theorem answers the converse question, namely, if the first eigenvalue of the sub-Laplacian takes its lower bound, then the quaternionic contact manifold is isometrical to the 3-Sasakian sphere [3].

\section{References}

[1] Ivanov, S., Petkov, A., Vassilev, D.: The sharp lower bound of the first eigenvalue of the sub-Laplacian on a quaternionic contact manifold. J. Geometric Anal., pp. 1-23. (2012). doi:10.1007/s12220-012-9354-9.

[2] Ivanov, S., Petkov, A., Vassilev, D.: The sharp lower bound of the first eigenvalue of the sub-Laplacian on a quaternionic contact manifold in dimension seven. Accept. Nonlinear Anal. arXiv:1210.6932 [math.DG].

[3] Ivanov, S., Petkov, A., Vassilev, D.: The Obata sphere theorems on a quaternionic contact manifold of dimension bigger than seven. arXiv:1303.0409 [math.DG].

Galina Panayotova (University of Library and Information Technology, Sofia, Bulgaria)

\section{Geometric Transformations in Computational Geometry}

In the paper are presented several geometric transformations: translation, rotation, scaling, axial symmetry, zoom in matrix type and homogeneous coordinates. Matrix representation is convenient for the purposes of geometric modeling. Those transformations can be applied for solve problems in computer graphics, design, for the purposes of visualization etc.

Mahfouz Rostamzadeh (Isfahan University of Technology, Isfahan, Iran)

Silvia Pianta (Università Cattolica, Brescia, Italy)

Sayed-Ghahreman Taherian (Isfahan University of Technology, Isfahan, Iran)

Helmut Karzel (TU München, Germany)

\section{Quasiparallism in General Absolute Planes}

Two lines $A, B$ of a general absolute plane $\mathcal{A}=(E, \mathfrak{G}, \alpha, \equiv)$ shall be called quasi-parallel - denoted by $A \ddagger B$-if $A \cap B=\emptyset$ and if $A$ and $B$ do not have a common perpendicular line. If $A \ddagger B$ then the pencil $\overbrace{A, B}$ is called quasi-end. 
Let $\omega(A)$ be the cardinal number of all quasi-ends which are incident with the line $A$. Since to any two lines $A, B \in \mathfrak{G}$ there is a motion mapping $A$ onto $B$ we have $\omega(A)=\omega(B)$ and therefore the cardinal number $\omega_{\mathcal{A}}:=\omega(A)$ is a characteristic feature for the absolute plane $\mathcal{A}$.

If $\mathcal{A}$ is a hyperbolic plane then two lines are quasi-parallel if they are hyperbolic parallel and so $\omega_{\mathcal{A}}=2$. We show, if $\mathcal{A}$ is a singular absolute but not an Euclidean plane then to each pair $(p, G)$ consisting of a line $G$ and a point $p$ with $p \notin G$ there are infinite many lines through $p$ which are quasi-parallel to the line $G$ hence $\omega_{\mathcal{A}}$ is an infinite cardinal number. The same is true if $\mathcal{A}$ is an absolute plane with elliptic congruence.

\section{Assia Rousseva (Sofia University, Bulgaria) \\ Ivan Landjev (New Bulgarian University, Sofia, Bulgaria)}

\section{On the Extendability of Arcs in Finite Projective Spaces}

Let $K$ be an $(n, w)$-arc in $\Sigma=\operatorname{PG}(k-1, q)$. Assume that for any hyperplane $H$ of $\Sigma$ we have one of

$$
K(H) \equiv n, n+1, \ldots, n+t \quad(\bmod q),
$$

for some integer $t, t<q$. Define an arc $\bar{K}$ in the dual geometry $\bar{\Sigma}$ by

$$
\bar{K}:\left\{\begin{array}{l}
\mathcal{H} \rightarrow \mathbb{N}_{0} \\
H \rightarrow \bar{K}(H):=n+t-K(H) \quad(\bmod q),
\end{array}\right.
$$

where $\mathcal{H}$ denotes the set of all hyperplanes in $\Sigma$. The following theorem is a sufficient condition for the extendability of $K$.

Theorem 1. Let $K$ be an $(n, w)$-arc in $\Sigma=\mathrm{PG}(k-1, q)$ and let $\bar{\Sigma}$ contain a hyperplane $H^{*}$ with $\bar{K}\left(x^{*}\right) \geq a>0$, a an integer, for all points $x^{*}$ incident with $H^{*}$. Then the arc $K$ is a-extendable, there exists an extension $K^{\prime}$ of $K$ with parameters $(n+a, w)$.

By the above theorem the extendability of $K$ depends on the structure of the $\operatorname{arc} \bar{K}$. It turns out that the arc $\bar{K}$ is divisible.

Theorem 2. For every subspace $S^{*}$ of $\bar{\Sigma}$ with $\operatorname{dim} S^{*} \geq 1$ :

$$
\bar{K}\left(S^{*}\right) \equiv t \quad(\bmod q) .
$$

In particular, $\bar{K}\left(\overline{\Sigma^{*}}\right) \equiv t(\bmod q)$.

By the above theorem, the arc $\bar{K}$ has the following properties:

1. the multiplicity of each point is at most $t$;

2. the multiplicity of each subspace of dimension $r, 1 \leq r \leq k-1$, is at least $t v_{r}$. 
Maruta [1] found all possible spectra for $\bar{K}$ in the case when $t=2$ and $q$ is an odd prime. In this case, all arcs with the above properties turn out to be extendable.

In this talk, we prove the following theorem that relates the spectrum of maximal hyperplanes to the extendability of Griesmer arcs.

Theorem 3. Consider a Griesmer $(n, w)$-arc $K$ with $K(H) \equiv n, n+1, \ldots, n+t$ $(\bmod q)$ for all hyperplanes $H$. Denote by $\left(a_{i}\right)$ the spectrum of the arc $\left.K\right|_{H}$, where $H$ is a hyperplane of multiplicity $w$, with respect to $K$. Let $A$ be the largest integer such that a $\left(t v_{k-1}+A, t v_{k-2}\right)$-minihyper contains a hyperplane in its support. If

$$
q a_{w-\lceil d / q\rceil-1}+2 q a_{w-\lceil d / q\rceil-2}+\cdots+(t-1) q a_{w-\lceil d / q\rceil-t+1} \leq A,
$$

then $K$ is extendable.

\section{Reference}

[1] Maruta, T.: A new extension theorem for linear codes, Finite Fields Appl. 10, 674-685 (2004)

\section{Arif A. Salimov (Ataturk University, Turkey) Rabia Cakan (Ataturk University, Turkey)}

\section{Musical Isomorphisms and Problems of Lifts}

Let $(M, g)$ be a smooth pseudo-Riemannian manifold of dimension $n$. A very important feature of any pseudo-Riemannian metric $g$ is that it provides musical (natural) isomorphisms $g^{b}: T M \rightarrow T^{*} M$ and $g^{\sharp}: T^{*} M \rightarrow T M$ between the tangent and cotangent bundles. We denote by $\mathfrak{T}_{q}^{p}(M)$ the set of all differentiable tensor fields of type $(p, q)$ on $M$. Let ${ }^{C} X_{T} \in \mathfrak{T}_{0}^{1}(T M),{ }^{C} \varphi_{T} \in \mathfrak{T}_{1}^{1}(T M)$ and ${ }^{C} S_{T} \in \mathfrak{T}_{2}^{1}(T M)$ be complete lifts of tensor fields $X \in \mathfrak{T}_{0}^{1}(M), \varphi \in \mathfrak{T}_{1}^{1}(M)$ and $S \in \mathfrak{T}_{2}^{1}(M)$ to the tangents bundle $T M$. In this paper, we transfer via the differential $g_{*}^{\text {b }}$ the complete $\operatorname{lifts}{ }^{C} X_{T} \in \mathfrak{T}_{0}^{1}(T M),{ }^{C} \varphi_{T} \in \mathfrak{T}_{1}^{1}(T M)$ and ${ }^{C} S_{T} \in \mathfrak{T}_{2}^{1}(T M)$ on the cotangent bundle $T^{*} M$. The transferred lifts $g_{*}^{b}{ }^{C} X_{T}, g_{*}^{b}{ }^{C} \varphi_{T}$ and $g_{*}^{b}{ }^{C} S_{T}$ are compared with the complete lifts ${ }^{C} X_{T^{*}} \in$ $\mathfrak{T}_{0}^{1}\left(T^{*} M\right),{ }^{C} \varphi_{T^{*}} \in \mathfrak{T}_{1}^{1}\left(T^{*} M\right)$ and ${ }^{C} S_{T^{*}} \in \mathfrak{T}_{2}^{1}\left(T^{*} M\right)$ in the cotangent bundle, and we show that

(a) $\quad g_{*}^{b}{ }^{C} X_{T}={ }^{C} X_{T^{*}}$ if and only if the vector field $X$ is a Killing vector field;

(b) $g_{*}^{b}{ }^{C} \varphi_{T}={ }^{C} \varphi_{T^{*}}$ if and only if the triple $(M, g, \varphi), \varphi^{2}=-\operatorname{Id}_{M}$ is a Kähler-Norden manifold;

(c) $\quad g_{*}^{b}{ }^{C} S_{T}={ }^{C} S_{T^{*}}$ if and only if the metric $g$ satisfies the Yano-Ako equations.

This paper is supported by The Scientific and Technological Research Council of Turkey (TBAG 112T111). 


\section{Udo Simon (TU Berlin, Germany)}

\section{Parallel Hypersurfaces}

We investigate parallel hypersurfaces in the context of relative hypersurface geometry, in particular including the cases of Euclidean and Blaschke hypersurfaces. We describe the geometric relations between parallel hypersurfaces in terms of deformation operators, and we apply the results to the parallel deformation of special classes of hypersurfaces, e.g. quadrics and Weingarten hypersurfaces.

Joint work with Barbara Opozda, Krakow.

\section{Grozio Stanilov (University of Sofia, Bulgaria)}

\section{Toeplitz Geometry Over Vector Space Induced from his 5 Order Symmetric Matrices}

Using the Maple package with(LinearAlgebra) : 5 order Toeplitz symmetric matrix can be introduced in the following way:

V5 := <q, r,s,t,u>:T5:=ToeplitzMatrix(V5, 5, symmetric);

The set of these regular matrices is not a group. But we have proved the following

Theorem 1. There are exactly two largest subsets of the set of regular Toeplitz symmetric matrices which are groups. These subsets are:
I. T51:=eval (T5, $[\mathrm{u}=\mathrm{r}, \mathrm{t}=\mathrm{s}])$;
II. T52:=eval $(T 5,[u=-r, t=-s])$;

So these subsets induce Toeplitz geometries denoted correspondingly by $\mathbf{T G}_{1}$ and $\mathbf{T G}_{2}$. Now we give some facts for the first Toeplitz geometry. Introducing the matrix

p51:=eval (T51, [q=x, r=y, s=z]) ;

We calculate at once: $\mathrm{T} 51 \cdot \mathrm{p} 51=\mathrm{p} 51 . \mathrm{T} 51$.

It means the Toeplitz geometry $\mathbf{T G}_{1}$ is a commutative geometry. The elements of the product T51.p51 are:

$X=q x+2 r y+2 s z, \quad Y=r x+(q+s) y+r z, \quad Z=s x+(r+s) y+(q+r) z$.

These formulas can be considered as fundamental transformations in this geometry.

Theorem 2. The directions $x+2 y+2 z=0, x^{2}-y^{2}-z^{2}-x y-x z+3 y z=0$ are relative invariant directions in the vector space of vectors $(x, y, z)$. We can introduce common invariants of two vectors and a vector and a 3-dimensional 
affine linear sub manifold (plane), defined by $e=a x+b y+c z+d=0$, and common invariants of two planes.

Some remarks: Considering Toeplitz symmetric matrices over some odd dimensions $3,5,7, \ldots$, we find in any case two subsets which are groups, but in the even dimensions $2,4,6, \ldots$, we find in any case only one subset which is group. The important problem is: how big are the dimensions for which these facts hold.

\section{Marta Teofilova (University of Plovdiv, Bulgaria)}

\section{Curvature Properties of Normal Almost Contact Manifolds with $B$-Metric}

Almost contact manifolds with $B$-metric (also known as Norden metric) are smooth $(2 n+1)$-dimensional manifolds equipped with an almost contact structure $(\varphi, \xi, \eta)$ and a compatible indefinite metric $g$ of signature $(n, n+1)$. The geometry of these manifolds is a natural extension of the geometry of the almost complex manifolds with Norden metric $[2,3]$ in the case of an odd dimension.

A classification of the almost contact $B$-metric manifolds is introduced in [1]. This classification consists of eleven basic classes and is obtained with respect to the properties of the covariant derivatives of the structural tensors.

In this work, we focus our attention on the classes of almost contact $B$-metric manifolds which have zero Nijenhuis tensor, i.e. normal almost contact manifolds with $B$-metric. We study the properties of the curvature tensor and obtain relations involving some scalar invariants. By virtue of these relations, we give necessary and sufficient conditions for such manifolds to be of isotropic Kähler-type. Also, we pay attention to the so-called main classes, i.e. manifolds for which $\nabla \varphi$ is expressed explicitly by the structural tensors. To illustrate the obtained results, in the last section, we construct and study an example of a manifold on a Lie algebra. This manifold belongs to the direct sum of two main classes of normal almost contact manifolds with $B$-metric.

Partially supported by project NI13-FMI-002 of the Scientific Research Fund, University of Plovdiv, Bulgaria.

\section{References}

[1] Ganchev, G., Mihova, V., Gribachev, K.: Almost contact manifolds with B-metric. Math. Balk. N.S. 7(3-4), 261-276 (1993).

[2] Gribachev, K., Mekerov, D., Djelepov, G.: Generalized B-manifolds. Compt. Rend. Acad. Bulg. Sci. 38(3), 299-302 (1985).

[3] Norden, A.P.: On a class of four-dimensional A-spaces. Russ. Math. (Izv VUZ) 17(4), 145-157 (1960) (in Russian). 


\section{Momme Johs Thomsen (Helmut-Schmidt-Universität Hamburg, Germany)}

\section{Nearrings of Mappings on Groups}

Familiar mathematical structures are the endomorphism rings (End $G,+, \circ$ ) determined by a given abelian group $(G,+)$. Endomorphism rings i.e. subrings of rings of the form (End $G,+, \circ$ ) are very general because every ring can be embedded into a ring (End $G,+, \circ$ ) for a suitable abelian group $(G,+)$.

That the sum of two endomorphisms is always again an endomorphism requires the commutativity of the group operation. Thus for non-abelian groups endomorphism rings are not defined. A generalization which makes sense for every group is the following concept.

Definition. For a given group $(G, \cdot)$ let $(M(G),+, \circ)$ be the set of all mappings from $G$ to $G$ with pointwise addition and with composition of mappings as multiplication, i.e. with + and $\circ$, where for $\alpha, \beta \in \mathrm{M}(G)$

$$
\begin{aligned}
\alpha+\beta \text { is defined by } g(\alpha+\beta) & =g \alpha \cdot g \beta \quad \text { and } \\
\alpha \circ \beta \text { is defined by } g(\alpha \circ \beta) & =(g \alpha) \beta \quad \text { for } g \in G \text {. }
\end{aligned}
$$

Then $(M(G),+, \circ)$ is a nearring in the sense of the following definition.

Definition. A nearring is a triple $(N,+, \cdot)$, where $N$ is a set and where + and - are binary operations on $N$, such that

i) $(N,+)$ is a group,

ii) $(N, \cdot)$ is a semigroup,

iii) $\quad c \cdot(a+b)=c \cdot a+c \cdot b$ for all $a, b, c \in N$.

Thus nearrings satisfy all the axioms a ring satisfies except for two: commutativity of addition and one of the distributive laws.

We are using the notation as explained in [8] or [6], [7], where also more details can be found. Much more material are in the four books now available and written by the authors Clay [1], Cotti Ferrero and Ferrero [2], Meldrum [4], Pilz [5].

The nearring $(M(G),+, \circ)$ is called the full transformation nearring on $G$. This nearring has the identity function $\operatorname{id}_{G}$ on $G$ as multiplicative identity. In general, a transformation nearring on $G$ is defined to be a subnearring of the full transformation nearring on $G$.

Transformation nearrings are very general because every nearring can be embedded into a nearring of the form $(M(G),+, \circ)$ for a suitable group $(G, \cdot)$.

Every nearring $(N,+, \cdot)$ has as subnearrings the so-called zero-symmetric part

$$
N_{0}:=\{n \in N \mid 0 \cdot n=n\}
$$

and the so-called constant part

$$
N_{c}:=\{n \in N \mid 0 \cdot n=0\} .
$$


For transformation nearrings $(N(G),+, \circ)$ on $G$ one has

$$
\begin{aligned}
& N_{0}(G):=\left(N(G)_{0}=\{\beta \in M(G) \mid 1 \beta=1\} \quad\right. \text { and } \\
& N_{c}(G):=\left(N(G)_{c}=\{\beta \in M(G) \mid 1 \beta=g \beta \quad \forall g \in G\} .\right.
\end{aligned}
$$

Besides $\left(M_{0}(G),+, \circ\right)$ and $\left(M_{c}(G),+, \circ\right)$ we want to consider further transformation nearrings on $G$, whose structure is more closely related to that of the group $(G, \cdot)$.

For a subset $S \subseteq M(G)$ let $(\mathrm{Ng}\langle S\rangle,+, \circ)$ be the subnearring of $(M(G),+, \circ)$ generated by $S$. We define seven transformation nearrings on $G$ by choosing generating sets $S \subseteq M(G)$ according to the following table. Here as usual

$$
\begin{aligned}
& \text { Inn } G=\{\alpha \in M(G) \mid \alpha \text { inner automorphism of } G\} \\
& \text { Aut } G=\{\alpha \in M(G) \mid \alpha \text { automorphism of } G\} \\
& \text { End } G=\{\alpha \in M(G) \mid \alpha \text { endomorphism of } G\} .
\end{aligned}
$$

\begin{tabular}{c|cccc}
$S$ & $\left\{\operatorname{id}_{G}\right\}$ & $\operatorname{Inn} G$ & Aut $G$ & End $G$ \\
\hline $\operatorname{Ng}\langle S\rangle$ & $P(G)$ & $I(G)$ & $A(G)$ & $E(G)$ \\
$S$ & & $\operatorname{Inn} G \cup M_{c}$ & Aut $G \cup M_{c}$ & $\operatorname{End} G \cup M_{c}$ \\
\hline $\operatorname{Ng}\langle S\rangle$ & $I C(G)$ & $A C(G)$ & $E C(G)$
\end{tabular}

I.e. for example $(I C(G),+, \circ)=\left(\mathrm{Ng}\left\langle\operatorname{Inn} G \cup M_{c}(G)\right\rangle,+, \circ\right)$. This nearring is called the nearring of (unary) polynomial functions on $G$.

Other names are

$$
P(G) \text { prime ring on } G
$$

$I(G)$ inner automorphism nearring on $G$

$A(G)$ automorphism nearring on $G$

$E(G)$ (full) endomorphism nearring on $G$.

It is clear that $\left(\alpha_{1}\right.$ being the additive identity of $\left.(M(G),+, \circ)\right)$

$$
\begin{array}{ccccc}
\left\{\alpha_{1}\right\} \leq P(G) \leq I(G) & \leq A(G) \leq E(G) & \leq M_{0}(G) \\
\leq & \leq & \leq & \leq & \leq \\
M_{c}(G) \leq & \leq I C(G) \leq A C(G) \leq E C(G) \leq M(G)
\end{array}
$$

where $\leq$ means here "is a subnearring of".

For $a \in G$ let $r_{a} \in M(G) \quad\left(l_{a} \in M(G)\right)$ be the right (left) translation of $G$ defined by $a$ (i.e. $x r_{a}=x \cdot a$ and $x l_{a}=a \cdot x$ for $x \in G$ ).

Let $T_{r}:=\left\{r_{a} \mid a \in G\right\} \subseteq M(G)\left(T_{l}:=\left\{l_{a} \mid a \in G\right\} \subseteq M(G)\right)$ be the set of all right (left) translations.

Then we have

$\left.(I C(G),+, \circ)=\left(\operatorname{Ng}\left\langle T_{r}\right\rangle,+, \circ\right)=\left(\operatorname{Ng}\left\langle T_{l}\right\rangle,+, \circ\right)=\operatorname{Ng}\left\langle\left\{\operatorname{id}_{G}\right\} \cup M_{c}(G)\right\rangle,+, \circ\right)$. 
For a subset $S \subseteq M(G)$ let $(\operatorname{Gp}\langle S\rangle,+)$ be the subgroup of $(M(G),+)$ generated by $S$. Each generating set we used is a subsemigroup $(S, \circ)$ of $(M(G)$, ०) consisting of rather special elements of $M(G)$. This fact can be used to show $\mathrm{Ng}\langle S\rangle=\operatorname{Gp}\langle S\rangle$ which furthermore implies:

For each of the eleven nearrings $N(G)$ in $(*)$ we have:

If $N(G)$ is in row $i(i=1$ or $i=2)$ then the constant part $N_{c}(G)$ is the first member in row $i$. If $N(G)$ is in column $j(1 \leq j \leq 6)$ then the zero-symmetric part $N_{0}(G)$ is the first member in column $j$; i.e. for example $I C_{c}(G)=M_{c}(G)$ and $I C_{0}(G)=I(G)$.

We have now set the scene such that many problems can be formulated. To explain what we mean by this we mention one of the first impressive results in nearring theory, now just 50 years old. In 1958 Fröhlich [3] showed that a finite nonabelian group $(G, \cdot)$ is simple if and only if $I C(G)=M(G)$, in other words if and only if the order of the polynomial nearring is as large as possible i.e.

$$
|I C(G)|=|G|^{|G|} .
$$

The following problems concerning our just defined transformation nearrings can be considered:

- Describe their structure (depending on the structure of the group $(G, \cdot)$ ).

- Give necessary and, or sufficient conditions that some of them coincide.

- Give formulas for their orders when the group $(G, \cdot)$ is finite.

We will give some results in this problem area.

\section{References}

[1] Clay, J.R.: Nearrings: Geneses and Applications. Oxford University Press, Oxford (1992).

[2] Cotti Ferrero, C., Ferrero, G.: Nearrings: Some Developments Linked to Semigroups and Groups. Kluwer Academic Publishers, Dordrecht (2002).

[3] Fröhlich, A.: The near-ring generated by the inner automorphisms of a finite simple group. J. Lond. Math. Soc. 33, 95-107 (1958).

[4] Meldrum, J.D.P.: Near-rings and their links with groups In: Research Notes in Mathematics, vol. 134. Pitman Advanced Publishing Program, Boston (1985)

[5] Pilz, G.: Near-rings. North-Holland/American Elsevier, Amsterdam (1977) (revised edition 1983).

[6] Saad, G., Syskin, S.A., Thomsen, M.J.: Endomorphism nearrings on finite groups, a report. In: Fong, Y., et al.: (eds.), Near-Rings and Near-Fields, pp. 227-238. Kluwer Academic Publishers, Dordrecht (1995).

[7] Saad, G., Syskin, S.A., Thomsen, M.J.: The inner automorphism nearrings $I(G)$ on all nonabelian groups $G$ of order $|G| \leq 100$. In: Saad, G., Thomsen, M.J. (eds.) Nearrings, Nearfields and K-Loops, pp. 377-402. Kluwer Academic Publishers, Dordrecht (1997).

[8] Saad, G., Thomsen, M.J.: Endomorphism nearrings: foundations, problems and recent results. Discret. Math. 208/209, 507-527 (1999). 
Constantin Udrişte (University Politehnica of Bucharest, Romania)

\section{Optimal Control on Tzitzeica Surfaces}

Tzitzeica surfaces are described as solutions of a system of partial differential equations, whose coefficients are constrained by a partial differential equation of hyperbolic type. We use these data, in combination with multitime optimal control to obtain information not only occur in the geometry of these surfaces. The moral is that multitime optimal control theory grafted on differential geometry problems produce new results.

\section{Reference}

[1] Arsinte, V., Udrişte, C.: Two-time optimal control with Tzitzeica PDEs constraints (unpublished).

Asen Velchev (UNWE Sofia, Bulgaria)

\section{Introducing Bachelors, Studying for Math Teachers, with Computer-Aided Graphical Solving and Exploration of Equations}

Graphs give intuitive and complete overall picture of the behavior of functions: increasing, decreasing, convexity, asymptotes, intersection points, etc. Therefore graphs are used in many branches: economy, politics, meteorology, engineering, biology, etc. The use of graphical methods and graphical solving of equations with a computer is strategic continuation of graphical approach. Another benefit from graphical solving (with computer) is the wide range solvable equations. It is hard or impossible to solve by standard methods equations like $x^{2}+\sqrt{7} x+\sqrt{3}=0, x^{3}+1,83 x+0,86-e=0,143 x^{3}-$ $149 x^{2}+11,933 x+811=0$ and $1.01 x^{3}-x^{2}+\sqrt{7} x+\sqrt{3}=0$, where coefficients are not small enough whole numbers. Other computer-solvable equations are mixed type ones: $x^{2}+x=\ln x, x=\sin x, \ln \left(x^{2}-x\right)=\tan x$, etc. Mixed here means polynomial-logarithmic, logarithmic-trigonometric, etc. All these equations cannot be solved graphically by paper and pen. A colleague with $\mathrm{PhD}$ in physics said that equations obtained in his practice are very rarely solvable by traditional school means and the most - by computer. Hence its perspective to introduce to the future math teachers computer aided graphical solving, problems related to it and didactic technologies to introduce it to school students.

When we solve graphically equations of the type $f(x)=g(x)$ or $F(x)=0$, there might occur several problems, considered in the paper. Future teachers have to know about these difficulties and we have introduced them with. Most important and frequent among these problems are: 
1. The graph of $F(x)$ may not appear on the screen or not all the equations roots may appear on it. First-case-example in our lecture was the equation $x^{2}-4 x-192=0$. It seemed like the computer did not respond. Our second-case-examples were: $x=5 \ln x$ and $x^{3}-18 x^{2}+17 x+240=0$. The solution in these cases is to zoom out until enough from the graph appear on the screen. It seemed there are no other solutions, as (according to the best student): "The one graph goes upwards, and the other-downwards." The concavity of logarithm curve helped us to prognose eventual second root and no more ones.

2. The graph of $F(x)$ may not be relevant, if the function $F(x)$ is not standard. Replacing the equation $F(x)=0$ with convenient equivalent one in the form $f(x)=g(x)$ usually removes this problem. Example: $\tan x-x^{2}=0$.

Even powerful SCA like MAPLE 11 err the graph of $y=\tan x-x^{2}$, as shown in the paper. The equation $\tan x-x^{2}=0$ is solvable in its equivalent form $\tan x=x^{2}$. Such examples show that the computer is not omnipotent. They are convenient for teachers to know about and show them to their students.

Getting ready graphs from the computer, the students can do different explanations, interpretations, suggestions, etc. I.e. they are not passive. Further, the dynamics geometry software GeoGebra, used by us, is convenient environment for experimentation. Exploration of parametric equations was another activity during our lesson. It is creative activity. The equation was $7 x^{k}+2 x^{3}-x+\sqrt{3 x^{2}+x}=7$ in our case. I reminded the students what means a term to be on negative/rational power.

After our experiment colleague-docent gave us positive overall estimation. The (university) students were interested during it and gave us positive estimation after it too.

Veselin Videv (Trakia University, Stara Zagora, Bulgaria)

Mariya Ivanova (University of Sofia, Bulgaria)

\section{Riemannian Manifolds Characterized by Stanilov Curvature Operators}

Let $(M, g)$ be an $n$-dimensional Riemannian manifold with a metric tensor $g$ and curvature tensor $R$, let $p$ be a point of $M$, and let $M_{p}$ be the tangent space to the manifold at this point. Then we consider the following curvature operators:

I. Jacobi operator:

$$
R_{X(u)}=R(u, X, X),
$$

defined for any unit tangent vector $X \in M_{p}$, at any point $p \in M$; 
II. Skew-symmetric operator:

$$
\kappa(u)=R(X, Y, u),
$$

defined for an arbitrary orthonormal basis $X, Y$ in a two-plane $\alpha \in M_{p}$, at a point $p \in M$;

III. Generalized Jacobi operator:

$$
\mathcal{R}_{E^{m}}=\sum_{i=1}^{m} R_{X_{i}}(u),
$$

defined for an arbitrary $m$-dimensional tangent subspace $E^{m} \in M_{p}$, at a point $p \in M$, where $\left\{X_{i}\right\}_{i=1, m}(m<n)$ is an orthonormal basis in $E^{m}$.

The Skew-symmetric operator and the Generalized Jacobi operator, defined in II and III by Stanilov, do not depend on any orthogonal transformation of their orthonormal basis in their inducing subspaces. Our results concerning the curvature operators are as follows:

Theorem 1. Let $(M, g)$ be an 4-dimensional Riemannian manifold. Then the following conditions are equivalent:

1. At any point $p \in M$, for any two-dimensional plane $\alpha \in M_{p}$ and its orthogonal complement $\alpha^{\perp} \in M_{p}$, holds:

$$
\kappa_{\alpha^{\perp}} \circ \mathcal{R}_{\alpha}=\mathcal{R}_{\alpha} \circ \kappa_{\alpha^{\perp}}
$$

2. At any point $p \in M$, for any two-dimensional plane $\alpha \in M_{p}$ and its orthogonal complement $\alpha^{\perp} \in M_{p}$, holds:

$$
\kappa_{\alpha} \circ \mathcal{R}_{\alpha}=\mathcal{R}_{\alpha} \circ \kappa_{\alpha}
$$

3. The Riemannian manifold $(M, g)$ is a space of constant sectional curvature.

Theorem 2. Let $(M, g)$ be an 4-dimensional Riemannian manifold, then the following conditions are equivalent:

1. At any point $p \in M$, for any two-dimensional plane $\alpha \in M_{p}$ and its orthogonal complement $\alpha^{\perp} \in M_{p}$ holds:

$$
\mathcal{R}_{\alpha^{\perp}} \circ \mathcal{R}_{\alpha}=\mathcal{R}_{\alpha} \circ \mathcal{R}_{\alpha^{\perp}}
$$

2. $(M, g)$ is an Einstein manifold.

Remark. Gilkey and Videv generalized these types of manifolds proving that condition 1 is equivalent to the equality:

$$
\rho \circ R=R \circ \rho
$$

where $\rho$ is the Ricci tensor and $R$ is the curvature tensor of the manifold and proving also that in $n$-dimensional reducible case they are direct products of an Einstein manifolds. 


\section{Dirk Windelberg (Leibniz University of Hannover, Germany)}

\section{Geometrical Descriptions of Statistical Clouds}

In this contribution, we are looking for a possibility to describe a "cloud" of individual (and not physical) reactions y (annoyance) on physically measured values $x$ (traffic noise).

- If a cloud has a visible "trend", then it may be functionally or causally.

- But if a cloud has not such a visible "trend", then statisticians are used to look for an "error of measurement" - but individual reactions are mostly stochastical and require unusual geometrical solutions:

In a first step we introduce a "cloud" of 12 relations between measured values and its reactions. We describe several different curves, where each of these should give a mathematical description of the 12 relations. Within the "cloud" of these relations between measured values and reactions we introduce additional a "confidence region", where a "boundary value" describes the highest level of measured value without being "highly annoyed".

In a second step we analyse the clouds of 1,300 dose-effect-relations of the German IF-Studie II and compare rail traffic as well as road traffic: We calculate and compare the boundary levels with the methods from the preceding parts.

\section{Elena Zizioli (University of Brescia, Italy)}

\section{Loops, Permutation Sets and Directed Graphs}

It is well known that if $P$ is a non-empty set, $\Gamma$ is a set of permutations acting regularly on $P$ and $o$ a distinguished point of $P$, then it is possible to equip $P$ with a loop operation with neutral element $o$ (see e.g. [1]). Conversely if $(P ;+)$ is a loop, it is possible to associate to $P$ a regular permutation set in several ways. Here, motivated by our work on slid product of loops, we firstly deepen the investigation of the relationships between regular permutation sets and loops, aiming at introducing a correspondence between properties of the loop structure and properties of regular permutation sets. In particular, since many different regular permutation sets can be associated to a loop, we characterize those regular permutation sets giving rise to the same loop, to isomorphic loops and to isotopic loops.

Moreover we introduce a correspondence between loops (and hence regular permutation sets) and directed graphs with a suitable edge colouring, generalizing a construction already known in the literature (see e.g. [2]), and we give a complete description of those graphs giving rise to the same loop, or to isomorphic and isotopic loops. Finally we relate some of the algebraic properties of the loop $(P ;+)$ to some configurations of the associated graph. 


\section{References}

[1] Karzel, H.: Loops related to geometric structures. Quasigr. Relat. Syst. 15, 47-76 (2007).

[2] Karzel, H., Pianta, S., Zizioli, E.: Loops, refection structures and graphs with parallelism. Results Math. 42(1-2), 74-80 (2002).

Received: October 23, 2013. 